\title{
Monodisperse Iron Oxide Nanoparticles by Thermal Decomposition: Elucidating Particle Formation by Second-Resolved in Situ Small- Angle X-ray Scattering
}

A. Lassenberger, ${ }^{\dagger, \perp}$ T. A. Grünewald, ${ }^{\ddagger} \perp$ P. D. J. van Oostrum, ${ }^{\dagger}$ H. Rennhofer, ${ }^{\ddagger}$ H. Amenitsch, ${ }^{\S}$ R. Zirbs, ${ }^{\dagger}$ H. C. Lichtenegger, $*,+$ and E. Reimhult* $*$ (†)

${ }^{\dagger}$ Department of Nanobiotechnology, Institute for Biologically Inspired Materials, University of Natural Resources and Life Sciences, Muthgasse 11, 1190 Vienna, Austria

${ }^{\ddagger}$ Department of Material Science and Process Engineering, Institute of Physics and Materials Science, University of Natural Resources and Life Sciences Peter-Jordan Strasse 82, 1190 Vienna, Austria

${ }^{\S}$ Institute for Inorganic Chemistry, Graz University of Technology, Stremayrgasse 9/V, 8010 Graz, Austria

Supporting Information

ABSTRACT: The synthesis of iron oxide nanoparticles (NPs) by thermal decomposition of iron precursors using oleic acid as surfactant has evolved to a state-of-the-art method to produce monodisperse, spherical NPs. The principles behind such monodisperse syntheses are well-known: the key is a separation between burst nucleation and growth phase, whereas the size of the population is set by the precursor-to-surfactant ratio. Here we follow the thermal decomposition of iron pentacarbonyl in the presence of oleic acid via in situ X-ray scattering. This method allows reaction kinetics and precursor states to be followed with high time resolution and statistical significance. Our investigation demonstrates that the final
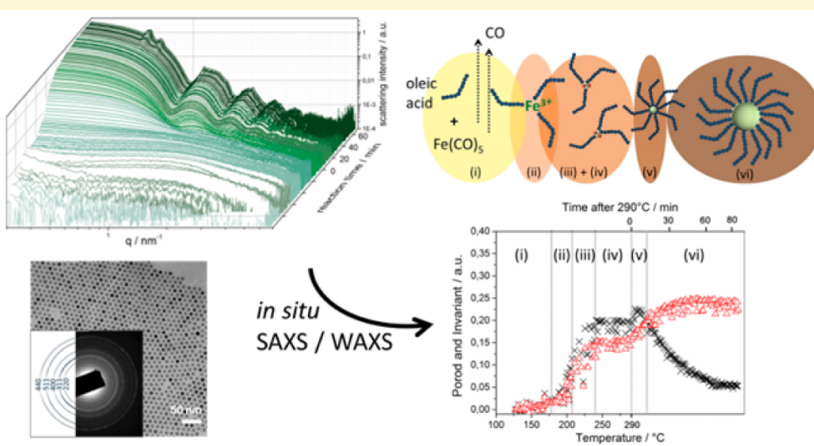
particle size is directly related to a phase of inorganic cluster formation that takes place between precursor decomposition and particle nucleation. The size and concentration of clusters were shown to be dependent on precursor-to-surfactant ratio and heating rate, which in turn led to differences in the onset of nucleation and concentration of nuclei after the burst nucleation phase. This first direct observation of prenucleation formation of inorganic and micellar structures in iron oxide nanoparticle synthesis by thermal decomposition likely has implications for synthesis of other NPs by similar routes.

\section{INTRODUCTION}

The synthesis of nanocrystals has taken a central place in inorganic chemistry for decades, but it is only in more recent years that the synthetic control has reached such precision that advanced nanotechnological applications have truly benefited. These applications range from quantum dots in conducting and optically active composite materials ${ }^{1}$ to mechanically reinforced scratch-resistant polymer nanocomposites ${ }^{2}$ and inorganic nanoparticles as markers and therapeutic tools in medicine ${ }^{3,4}$ and cosmetics. ${ }^{5}$ Low polydispersity and precise control over nanoparticle size and shape are required for these applications.

A decisive breakthrough in the synthesis of iron oxide nanoparticles was the work of Hyeon and co-workers, ${ }^{6}$ who pioneered the synthesis of monodisperse, spherical iron oxide nanoparticles (NPs). Several different routes building on thermal decomposition of metallo-organic precursors have been demonstrated to lead to the synthesis of nanoparticles with low polydispersity. ${ }^{7-10}$ The highest level of control demonstrated for this method has been in combination with ligand-assisted, for example, oleic acid-assisted, control over the growth of the NP core after nucleation. ${ }^{7,11,12}$ As first described by LaMer $^{13}$ and later by Talapin et al., ${ }^{14}$ the key to low polydispersity is to achieve effective separation of burst nucleation from particle growth. A uniform growth rate of the particles then results in a monodisperse nanoparticle population with the size set mainly by the molar precursor to ligand ratio.

Exquisite control over the nanoparticle size distribution has been verified in many publications, ${ }^{7,15-17}$ but time-resolved investigations of such burst nucleation and growth processes are almost absent. Studies have been limited to the extraction of samples for ex situ transmission electron microscopy (TEM) analysis $^{18}$ at low time resolution and absorption spectroscopy that can shed light on the chemical composition of the sample, also performed at low time resolution. ${ }^{19}$ There is, however, a solid theoretical foundation describing the growth of

Received: March 24, 2017

Revised: $\quad$ May 2, 2017

Published: May 2, 2017 
monodisperse nanoparticles, which has been used to interpret the experimental work on reaction kinetics that has been performed. This and related experimental work, relevant for our study, will be briefly recapitulated here.

Monodisperse nanoparticle or nanocrystal growth requires size focusing, although classical theory for nanoparticle growth predicts defocusing to occur over time through ripening. Already, the early LSW (Lifshitz and Slyozov ${ }^{20}$ and Wagner ${ }^{21}$ ) theory predicted that a unique shape of the size distribution for the late stages of growth can be obtained independent of the initial conditions. ${ }^{20}$ Talapin et al. later demonstrated with a more complete analysis that even if the nucleation and growth phases are not completely separated, for example, by the hot injection method, a much higher nucleation than growth rate will still lead to a similar outcome. ${ }^{14}$ For diffusion-limited particle growth, a narrow distribution was found in simulations to be increasingly skewed toward smaller size as a function of time. Reaction-limited growth results in much faster broadening and skewing of the distribution with a tail toward smaller size. These results lay the basis for extended LSW models used to explain synthesis of monodisperse nanoparticles such as quantum dots and superparamagnetic iron oxide nanoparticles (SPION). Several additional conclusions were drawn such as that for transport-limited growth, a lower transport coefficient (diffusion coefficient) leads to a lower limiting standard deviation of the particle size distribution. This distribution can be significantly narrower than for micron-sized particles. A higher surface tension yields a lower standard deviation, but originally a standard deviation of $15-20 \%$ regardless of nanoparticle size or initial size distribution was simulated. ${ }^{14}$ While the original LaMer-type model is not strictly applicable to growth of ligand-stabilized nanoparticles of size close to the nucleation size, extended kinetic models have been developed that are in excellent agreement with such growth. ${ }^{22}$

A slower growth rate yields crystal growth with fewer defects, that is, nanoparticles growing close to the critical size have the lowest growth rate and should therefore have higher crystallinity. ${ }^{23}$ Whether this should lead to more faceted or smoother crystals is debated, but the argument for the growth of stable facets is strong. ${ }^{23,24}$ A high supersaturation ratio leads to low critical size and thereby to more nuclei being above the critical size with positive growth rates. ${ }^{14}$

In hot-injection synthesis, the achievable very high supersaturation ratio yields a simple explanation for the separation of burst nucleation and growth phases. The argument for achieving extreme supersaturation is less clear for heat-up methods, ${ }^{18}$ but the very strong temperature dependence of the nucleation rate ${ }^{14}$ can be used to make a similar argument also for finite heating rates by the heat-up method. However, heatup methods have also been suggested to proceed through an initial decomposition of precursors to polyiron oxo clusters that serve as monomers for the nucleation and growth of iron oxide nanoparticles. ${ }^{25}$ Kwon et al. confirmed that decomposition of precursors in a heat-up process using iron oleate complex precursors did not result in immediate nucleation and growth of nanoparticles and therefore confirmed the existence of an intermediate monomer state argued to be polyiron oxo clusters. ${ }^{18}$ A similar intermediate is expected to play an important role in all metal-oleate heat-up methods. Sizeexclusion chromatography, seconded by TEM, and crystallization yield was used to monitor the size growth of nanoparticles after nucleation at minute time resolution. The nucleation, regarded as the conversion of monomers to nanocrystals, was followed only via the crystallization yield through elemental analysis, which only had $10 \mathrm{~min}$ resolution and therefore could not produce the detailed kinetics of this step. Nucleation was completed within 20 min after the critical temperature of $320{ }^{\circ} \mathrm{C}$ was reached and maintained. TEM images, however, indicated the presence of a bimodal distribution of nuclei and small nanoparticles within the first few minutes after the critical temperature was reached, where most nuclei kept a constant diameter of $\sim 1.7 \mathrm{~nm}$. After this time, the TEM analysis indicated that the nucleation was complete and growth ensued, which for the first few minutes served to smoothen (round) the shape and grow the NPs uniformly. It should be noted that the TEM images and histograms did not trace the shift of the predominant small nuclei to larger size, but rather seemed to indicate a sudden growth within the first 1-2 min in the population of the larger, 5-6 $\mathrm{nm}$ in diameter, nanoparticles, which then continuously grew to close to $10 \mathrm{~nm}$ in diameter. These sizes already correspond to quite large cores. At the final size, their shape reverted back to faceted, seemingly cubic, in correspondence to the lowest surface energy facets of magnetite and maghemite. The final step also corresponds to a broadening of the size distribution.

Given the gaps in the understanding of the steps leading up to nucleation and the lack of sufficient time resolution data for the nucleation and growth, the aim of our work is to extract the reaction kinetics and precursor states during the synthesis of size-focused, extremely monodisperse iron oxide nanoparticles with unprecedented time resolution and statistical significance of the measurements. By focusing on in situ detection of the formation of sample inhomogeneity, interpreted as micelles, clusters, nuclei, and particles, we link synthesis parameters to size distributions via clearly time-resolved kinetic intermediate states.

We investigate the synthesis of superparamagnetic iron oxide nanoparticles by thermal decomposition of iron(0)pentacarbonyl $\left(\mathrm{Fe}(\mathrm{CO})_{5}\right)$ in dioctyl ether in the presence of oleic acid at a defined ratio, following the method introduced by Hyeon and co-workers. ${ }^{26}$ By continuous online extraction of small fractions of sample during a running reaction, we follow the nucleation and growth using small-angle X-ray scattering (SAXS), wide-angle X-ray scattering (WAXS), and TEM practically in situ. While TEM can be used to determine the morphology of synthesized nanoparticles, its statistical relevance is poor. Furthermore, small clusters cannot be reliably imaged and metallo-organic precursor states such as micelles that are only stable in solution cannot be imaged at all under vacuum conditions. SAXS and WAXS can in this respect provide an invaluable tool to estimate the average size, crystallinity, amount of scattering material, internal surface area, ${ }^{27,28}$ and polydispersity of micelles, ${ }^{29}$ clusters, and particles $^{30}$ continuously throughout the synthesis. These methods have previously been used to elucidate the reaction kinetics for formation of, for example, Pt nanoparticles from intermediate precursor cluster states. ${ }^{22}$ Using in situ SAXS/ WAXS we could follow the entire formation of iron oleate complexes, further decomposition to polyiron oxo clusters serving as monomers for nanoparticle nucleation, as well as the nucleation and growth of iron oxide nanoparticles at a time resolution of seconds. The different phases could be clearly distinguished, which confirmed the modeling of nanoparticle synthesis by the heat-up method using the separation of burst nucleation and growth phases demonstrated for hot injection 
synthesis routes, ${ }^{31}$ but with added detail on crucial and largely unmapped intermediate phases. By this approach, we further investigated the influence of the heating rate and in particular the $\mathrm{Fe}(\mathrm{CO})_{5}$ /oleic acid ratio on particle growth kinetics and morphology.

\section{MATERIALS AND METHODS}

Reagents. Iron $(0)$ pentacarbonyl $\left(\mathrm{Fe}(\mathrm{CO})_{5}, 99,99 \%\right.$ trace metal basis), oleic acid (OA, $\geq 93 \%$ technical grade), and dioctyl ether $\left(\mathrm{Oct}_{2} \mathrm{O},>99 \%\right)$ were purchased from Sigma-Aldrich; ethanol (EtOH $>96 \%$ technical grade) and toluene ( $\geq 95 \%$ Rotipuran) were obtained from Carl Roth.

All chemicals were used as received without further purification.

Core Synthesis. Superparamagnetic oleic acid stabilized iron oxide nanoparticles were synthesized by thermal decomposition of $\mathrm{Fe}(\mathrm{CO})_{5}$ in the presence of oleic acid as capping agent according to a slightly modified heat-up procedure previously described by Hyeon et al. ${ }^{26}$ To investigate the kinetics of this synthesis route, the amounts of solvent $(50 \mathrm{~mL})$ and precursor $(1 \mathrm{~mL})$ were kept constant and either the amount of oleic acid or the heating rate was varied (see Table 1 for

\section{Table 1. Molar Ratios for NPs of Different Size ${ }^{a}$}

\begin{tabular}{lcc}
\multicolumn{1}{c}{$\mathrm{NP}$ size } & $\mathrm{Fe}(\mathrm{CO})_{5} / \mathrm{OA}($ molar ratio) & heating rates $[\mathrm{K} / \mathrm{min}]$ \\
Small $(3-4 \mathrm{~nm})$ & $1: 1.7$ & 3,9 \\
Medium $(5-6 \mathrm{~nm})$ & $1: 1.9$ & 3 \\
Large $(9-10 \mathrm{~nm})$ & $1: 3.0$ & $1,3,9$
\end{tabular}

${ }^{a}$ Experimental details for the NP synthesis experiments where the size was varied by $\mathrm{Fe}(\mathrm{CO})_{5} / \mathrm{OA}$ ratio and uniformity by heating rate.

experimental details). Typically, a mixture of $50 \mathrm{~mL}$ of $\mathrm{Oct}_{2} \mathrm{O}$ and the respective amount of oleic acid $(7.06 \mathrm{~mL}$ of OA for "Large", $4.48 \mathrm{~mL}$ of OA for "Medium", and $3.87 \mathrm{~mL}$ of OA for "Small" cores) was heated with a ramp of $10 \mathrm{~K} / \mathrm{min}$ to $100{ }^{\circ} \mathrm{C}$ under a constant flow of $\mathrm{N}_{2}$ and held at $100{ }^{\circ} \mathrm{C}$ for $10 \mathrm{~min}$ to remove $\mathrm{H}_{2} \mathrm{O}$. One milliliter of $\mathrm{Fe}(\mathrm{CO})_{5}$ was injected rapidly, and the reaction mixture was heated to reflux $\left(290{ }^{\circ} \mathrm{C}\right)$ with a temperature ramp of $3 \mathrm{~K} / \mathrm{min}$ and held at this temperature for $1 \mathrm{~h}$. Samples were continuously removed by a pump and autosampler and collected as 80 fractions per synthesis. The fractions were allowed to cool to room temperature. For TEM analysis, the NPs were precipitated twice with EtOH and dispersed in $200 \mu \mathrm{L}$ of toluene.

A schematic of the experimental setup is given in Figure 1. The Xray sample cell consisted of a Kapton tube with an inner diameter (ID) of $1.64 \mathrm{~mm}$ and a wall thickness of $0.03 \mathrm{~mm}$ (Detakta $\mathrm{GmbH}$, Norderstedt) and connected to the reaction flask and autosampler by Teflon tubing (BOLA) with an ID of $1 \mathrm{~mm}$.

A peristaltic pump, High Precision Multichannel Dispenser (ISM935 IPC IDEX Ismatec), was used to pump the solution out of the reaction vessel. The tube for the peristaltic pump was made of Tygon R367 (IDEX Ismatec) with an ID of $0.64 \mathrm{~mm}$ and wall thickness of $0.91 \mathrm{~mm}$. The flow velocity of the pump was calibrated prior to each experimental run and was kept constant at $100 \mu \mathrm{L} / \mathrm{min}$ for heating rates of 3 and $9 \mathrm{~K} / \mathrm{min}$ and at $54.9 \mu \mathrm{L} / \mathrm{min}$ for the $1 \mathrm{~K} /$ min heating rate. A camera was installed to count droplets in $10 \mathrm{~min}$ intervals to monitor the flux. The outlet fractions were collected using a BioRad 2110 HPLC fraction collector autosampler with an integration time of $5 \mathrm{~min}$ for all experiments except for "Large" cores $(3 \mathrm{~K} / \mathrm{min})$ where an integration time of $1.5 \mathrm{~min}$ was set. The outlet to the autosampler was made of a stainless steel tube with an ID of $1 \mathrm{~mm}$.

To match the temperature in the reaction vessel with the SAXS/ WAXS signal and the fractions of the autosampler, the time lag between the extraction of a sample and the measurement at the X-ray window was considered.

Small Angle X-ray Scattering. Combined in situ SAXS/WAXS measurements were carried out at the SAXS beamline (5.2L) of the synchrotron light source Elettra, Trieste, Italy, operated at $2 \mathrm{GeV}$ and

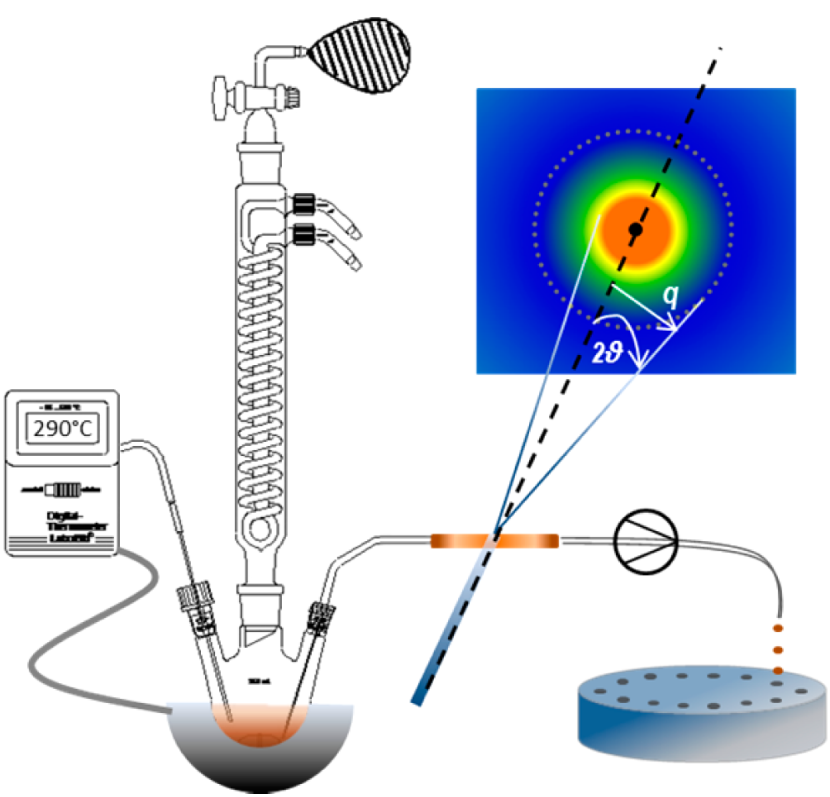

Figure 1. Schematic of the experimental setup. The water-cooled reflux aperture was heated by an electronically coupled heating mantle. The sample was continuously pumped from the reaction mixture by a peristaltic pump through the X-ray transparent Kapton sample chamber and sampled by an autosampler.

$300 \mathrm{~mA}$ ring current. ${ }^{32}$ The experiment was carried out at a photon energy of $8 \mathrm{keV}$, with simultaneous acquisition of the SAXS signal with a Pilatus3 $1 \mathrm{M}$ detector and WAXS signal with a Pilatus $100 \mathrm{~K}$ detector. The SAXS sample to detector distance of $1000 \mathrm{~mm}$ gave access to a $q$-range from $0.13-6.5 \mathrm{~nm}^{-1}$, and the WAXS detector setting gave access to scattering vectors from $16.5-26 \mathrm{~nm}^{-1}$. The two setups were calibrated with the standards silver behenate and $p$-bromo benzoic acid, respectively.

To check for radiation damage, a test run with an intermittent pumping scheme was carried out which aimed at replacing the complete volume in the tube with fresh material from the reaction vessel and taking consecutive frames with $0.5 \mathrm{~s}$ exposure time and $10 \mathrm{~s}$ total frame time for $2 \mathrm{~min}$ in 29 pumping cycles. Within this time span, no degradation of the particles could be detected, and the effect of radiation damage on the sample could therefore be neglected for the continuously analyzed samples. The evaluated size and polydispersity can be seen in Figures S4 and S5.

Data were acquired with a $0.5 \mathrm{~s}$ time increment and integrated azimuthally using the Fit2D data package. The WAXS data have been averaged over 10 frames, giving a time resolution of $5 \mathrm{~s}$, and the SAXS data were processed with $0.5 \mathrm{~s}$ time resolution. The reaction mixture at $100{ }^{\circ} \mathrm{C}$ at the beginning of the experiment was used to record the background that was subtracted from the SAXS and WAXS data.

Data Treatment. The WAXS data of the magnetite (311) reflection were fitted with a Gaussian function, and its full-width at half-maximum (fwhm) was used to calculate the crystallite size $d_{\mathrm{XRD}}$ according to the Scherrer relationship:

$$
d_{\mathrm{XRD}}=\frac{K \lambda}{\Delta(2 \theta) \times \cos \theta}
$$

where $K$ is the Scherrer prefactor ( 0.9082 in case of the (311) reflection in a cubic crystal system $\left.{ }^{33}\right), \lambda$ the wavelength of the incident $\mathrm{X}$-rays, $\Delta(2 \theta)$ is the fwhm of the diffraction peak, and $\theta$ is half the scattering angle.

The intensity of the SAXS signal $I(q)$ can generally be described as

$$
I(q) \propto|F(q)|^{2} \times S(q)
$$

where $F(q)$ is the form factor of the scattering signal, $S(q)$ the structure factor of the particle-particle interaction, and $q$ the 
scattering vector. The contribution of the structure factor only influences the detected signal if the concentration of the particles is high enough to create short-range order. This contribution was only detected when particles accumulated on the capillary wall at the end of the synthesis and was hence disregarded.

The form factor was described by the scattering of a sphere with radius $R$ as

$$
F(q, R)=\frac{3(\sin (q R)-q R \cos (q R)}{(q R)^{3}}
$$

To account for the polydispersity of the system, a Schultz distribution of the radii of the cores was introduced. ${ }^{34}$ All data treatment was carried out in Mathematica 9.0.1. Fitting was carried out using a Levenberg-Marquardt algorithm minimizing fit residuals. ${ }^{35,36} \mathrm{~A}$ form factor could only be fitted to the data after nucleation, after which it was used to determine growth and polydispersity of the particle size.

The Invariant and the Porod constant $P$ can also be used to describe the scattering signal. ${ }^{37}$ The Invariant describes the integral scattering intensity of the system and shows the total amount of scattering material, regardless of the structure of the scatterer. It depends on the volume fraction of the different phases and the scattering length density contrast, ${ }^{37,38}$ and it can be written as

$$
\text { Invariant }=\int_{0}^{\infty} q^{2} \times I(q) \times \mathrm{d} q
$$

At large $q$, the scattering curve can be described as

$$
I(q) \propto \frac{P}{q^{4}}
$$

in case of particles or structures with a smooth surface. $P$ is proportional to the total inner surface of the scattering system. The Invariant and the Porod constant were calculated throughout the core synthesis, as they do not require fitting of a model to features in the scattering curve.

Transmission Electron Microscopy (TEM). TEM studies were performed on a FEI Tecnai G2 20 transmission electron microscope operating at $120 \mathrm{kV}$ or $200 \mathrm{kV}$ for high resolution imaging. Samples were prepared by dropping dispersions of NPs in toluene onto a 300mesh carbon-coated copper grid and subsequently evaporating the solvent in air. Size distributions were evaluated using PEBBLES ${ }^{39}$ software package with a local intensity fitting algorithm. Approximately 800 NPs were sampled for each size determination by Pebbles.

\section{RESULTS}

Analysis of Kinetics of Synthesis From SAXS, WAXS, and TEM. We followed the evolution of the size and polydispersity of the NPs with subsecond time resolution during synthesis using SAXS/WAXS. Figure 2 shows the temporal evolution of the SAXS signal during the synthesis of the "Large" cores in Table 1 using a heating ramp of $3 \mathrm{~K} / \mathrm{min}$. The shoulder and the minima shift toward smaller $q$ with time, which is expected for NPs that increase in size. The raw SAXS signals also show increasing definition of multiple minima, which strongly indicates the increasing monodispersity of the system with time. Toward the end of the measurement, sharp peaks appear at $q=0.51 \mathrm{~nm}^{-1}$ and $q=0.60 \mathrm{~nm}^{-1}$. These peaks correspond to a face-centered-cubic superlattice of particles that forms due to the high monodispersity and concentration of NPs on the capillary wall in the measurement zone.

Figure 3 shows TEM micrographs taken at selected time intervals from the synthesis of the large NPs. At early times after nucleation, the particles appear smaller, less uniform, and more polydisperse than toward the end of the synthesis. The narrowing of the size distribution becomes evident from the assembly of regular superlattices of NPs in hexagonal order as the growth progresses. ${ }^{40}$ The electron diffraction pattern at $t=$

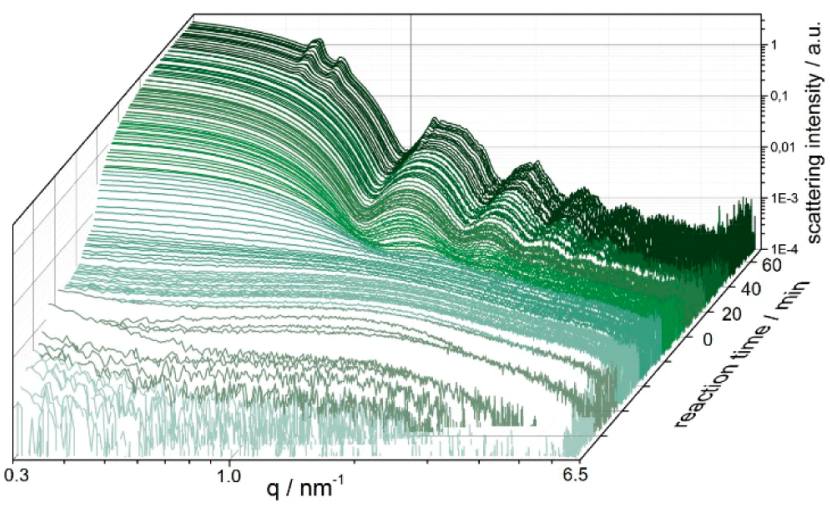

Figure 2. Scattering curves for synthesis of large NPs. Temporal evolution of the SAXS signals recorded continuously on the sample mixture for the synthesis of large NPs for a $q$-range from $0.3-6.5$ $\mathrm{nm}^{-1}$. During the heat up phase, the temperature is continuously increased and kept constant from $t=0$. The increasingly defined minima and final appearance of structure peaks corresponding to hexagonally ordered particles show the growth of the increasingly monodisperse nanoparticles.

$0 \mathrm{~min}$ is blurry, and no sign of crystallinity is observed. At $t=$ $12.4 \mathrm{~min}$, a diffraction pattern typical for maghemite or magnetite starts to form and becomes more pronounced as the NPs grow. The ratio of $d$-spacings of the obtained pattern is in good agreement with the JCPDS database numbers for magnetite or maghemite. ${ }^{41}$

The SAXS data were fitted with a form factor describing polydisperse spheres to extract the time evolution of the average radius and width of the particle size distribution during synthesis. ${ }^{34,38}$ Nucleation is expected to start when the reflux temperature of $290{ }^{\circ} \mathrm{C}$ is reached $(t=0)$ in Figure 2. Figure 4 shows that "Large" nanoparticles grew quickly in size $0.5 \mathrm{~min}$ after the final temperature was reached (red crosses) and proceeded until saturation, in the case of "Large" NPs at a radius of $4.5 \mathrm{~nm}$. As the particles start to grow, it is evident that they are highly polydisperse. The polydispersity is shown in Figure 4 (blue triangles). After peaking, as soon as the NPs have reached a size that can be determined by SAXS, the polydispersity quickly decreases to a very low value of $\sim 5 \%$ indicative of size focusing.

The growth of the iron oxide crystal size in the sample could be determined using the diffraction signal recorded by WAXS. Half the Scherrer width calculated from the WAXS signal is plotted in Figure 4 and compared to the particle radius fitted to the SAXS data. (see Figure S3 in the Supporting Information for WAXS data). There are no crystallites detected before particle growth commences. The formation of crystalline particles is seen $13.3 \mathrm{~min}$ after the final temperature is reached $(t=13.3$, Figure 4, black squares), which is in good agreement with the electron diffraction data (insets Figure 3). The WAXS data reveal a growth of the crystal size that is initiated after the start of growth of the iron oxide nanoparticles but that is faster than the particle growth. At the end of the particle growth, the crystal radius estimated from the Scherrer width is similar to the particle size. The observed small difference is likely related to the estimate of the Scherrer prefactor or to the different assumptions inherently made for polydispersity in the two respective models; the Scherrer width is only sensitive to the coherently scattering domains in the sample and does not take polydispersity into account, while the fit of the spherical form 

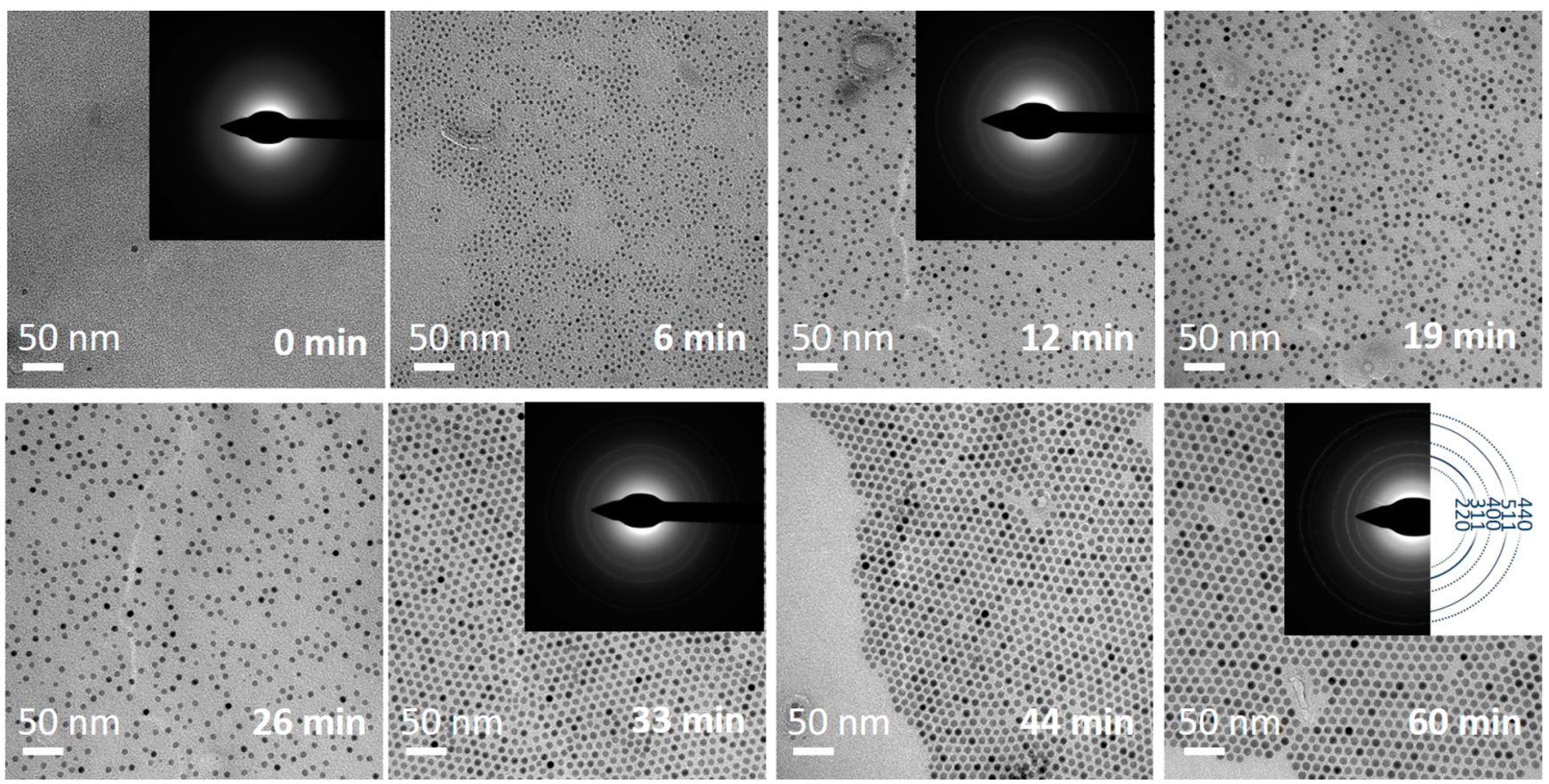

Figure 3. TEM micrographs and electron diffraction patterns of selected fractions for the synthesis of "Large" cores. The nanoparticles grow homogeneously, and superlattices are formed when the polydispersity of the nanoparticles approaches $5 \% .{ }^{40}$ The diffraction patterns show the increase in crystallinity with the reaction time and thus particle size.

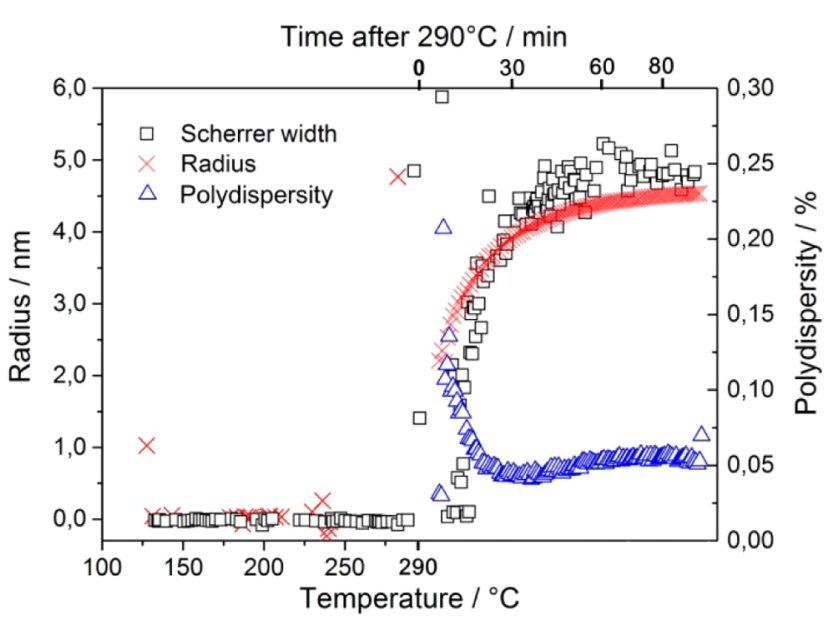

Figure 4. Time-evolution of radius from SAXS and WAXS data of "Large" core synthesis at $3 \mathrm{~K} / \mathrm{min}$. Extracted data for the nanoparticle radius (red crosses), polydispersity of the SAXS radius (blue triangles) and the crystallite radius determined as half the Scherrer width (black squares) as a function of time.

factor to the SAXS data explicitly allows for the polydispersity to be fitted.

Figure 5 shows the Invariant and the Porod constant for the data analyzed in Figure 4, corresponding to the synthesis of "Large" cores at $3 \mathrm{~K} / \mathrm{min}$ heating rate. This analysis provides valuable insights into the kinetics of precursor decomposition to form iron oleate complexes during the heat-up phase of the synthesis, followed by the formation of polyiron oxo clusters that act as precursors for the nanoparticle growth.

The Porod constant (black crosses) correlates with the inner surface of the system. It starts to increase at about $140{ }^{\circ} \mathrm{C}$, which we interpret as the onset of formation of the iron oleate complex since the temperature is too low for oxide particle

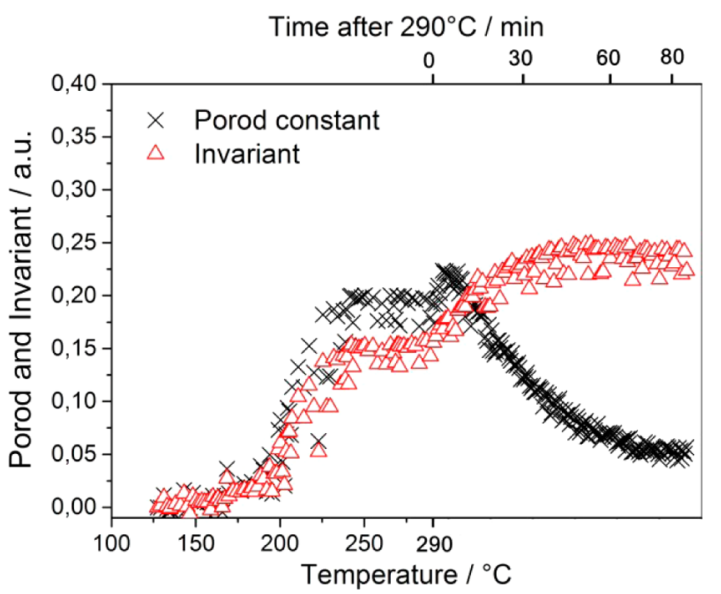

Figure 5. Time-evolution of Porod constant and Invariant for "Large" NP synthesis. The Porod constant (black crosses) and Invariant (red triangles) of the SAXS data recorded for "Large"-core synthesis heated at $3 \mathrm{~K} / \mathrm{min}$. The concerted increase during the heat-up phase corresponds to the formation of the iron oleate complex followed by burst nucleation of polyiron oxo clusters shortly after the final temperature of $290{ }^{\circ} \mathrm{C}$ is reached. These clusters are consumed as monomers for the core growth, which yields a decrease in Porod constant (internal area), while the Invariant increases during nanoparticle growth due to an increase in scattering contrast.

growth and the SAXS data does not reveal the formation of particles at this stage. Although the formation of particles is ruled out by the SAXS data (Figure 4), it is possible that a different precursor is already formed during this early part of the heat-up phase. Hyeon et al. have for example suggested that polyiron oxo clusters are formed from the oleate complex as the actual precursor for nanoparticle nucleation and growth. The concentration of this precursor reaches a stable plateau at about $240{ }^{\circ} \mathrm{C}$ in Figure 5 . 
The peak starting at $t=0.8 \mathrm{~min}$ with a maximum at $t=6.5$ min, shortly after the reflux temperature of $290{ }^{\circ} \mathrm{C}$ has been reached, indicates the burst nucleation. This is in good agreement with the onset of NP growth seen in Figure 4. The decay of inner surface thereafter shows the growth of the particles by consuming the precursor. Toward the end of the particle growth, the internal area as estimated by the Porod constant remains stable as the nanoparticles have reached their final size and the precursor has been depleted.

The Invariant (Figure 5, red triangles) is proportional to the total amount of scattering material irrespective of its size, but it is sensitive to the scattering contrast and concentration. The Invariant mimics the growth, plateau, and burst nucleation indicated by the Porod constant. After nucleation, however, the Invariant continuously increases, which indicates a further increase in the total amount of scattering material or in the contrast between the scattering centers and the complex organic solvent phase.

The Invariant would not increase if the particles only grow by consuming precursors formed during the heat-up phase since the total amount of scattering material would be constant. Thus, the increase in the Invariant should be attributed either to the creation of more scattering material from the OAcontaining medium or to an increase in the scattering contrast between the scattering centers and the solvent. The latter is possible in our system since the interpretation of the Invariant as being directly proportional to the amount of scattering material in the solution is strictly true only for a two-phase system; for a complex system as the one under study with at least three phases, the interface between the high electron density phase (iron complexes/clusters) and the low density organic solvent can change in density, extension, and composition. That the iron oleate complexes and precursor states are detectable through both the Invariant and the Porod constant implies that the complex fluid has structure. Otherwise, the fluid will not contain a phase boundary with sufficient scattering contrast. Most likely, a micellar structure of complexes or precursors with an oleate/oleic acid shell is present in the $\mathrm{Oct}_{2} \mathrm{O}$ with additional dissolved OA. It is this oleate/oleic acid shell that can potentially change in density, composition, and ordering such that it contributes more or less to the scattering signal.

Effect of $\mathrm{Fe}(\mathrm{CO})_{5} / \mathrm{OA}$ Ratio. The $\mathrm{Fe}(\mathrm{CO})_{5} / \mathrm{OA}$ ratio is known to control the size of the final nanoparticles. For the same concentration of $\mathrm{Fe}(\mathrm{CO})_{5}$, a decrease in the OA concentration is expected to yield smaller NPs. ${ }^{6}$ Figure 6 shows the evolution of size and polydispersity fitted from the SAXS data for synthesis of "Small", "Medium", and "Large" iron oxide nanoparticles by varying the $\mathrm{Fe}(\mathrm{CO})_{5} / \mathrm{OA}$ concentration according to Table 1 . The heating rate was in all cases kept at 3 $\mathrm{K} / \mathrm{min}$, stopping at $290{ }^{\circ} \mathrm{C}$, and holding the final temperature for $66 \mathrm{~min}$ (small and medium NPs) or $83 \mathrm{~min}$ (large NPs). A sharp increase in radius can be observed for all NP sizes, whereas the temperature for onset of this rapid growth decreases as the OA concentration decreases. For large particles (black circles), the growth starts at about $0.5 \mathrm{~min}$ after the final temperature is reached (cf. Table S1 in the Supporting Information for additional numerical parameters extracted from all reactions). This onset is lowered to $\sim 260{ }^{\circ} \mathrm{C}$ for midsized (red circles) and to $\sim 240-250{ }^{\circ} \mathrm{C}$ for small NPs (blue circles). The final size of NPs is reached faster for small NPs (6.5 min after reaching the final temperature) than for "Medium" NPs (9.6 min after reaching $290^{\circ} \mathrm{C}$ ) and "Large"

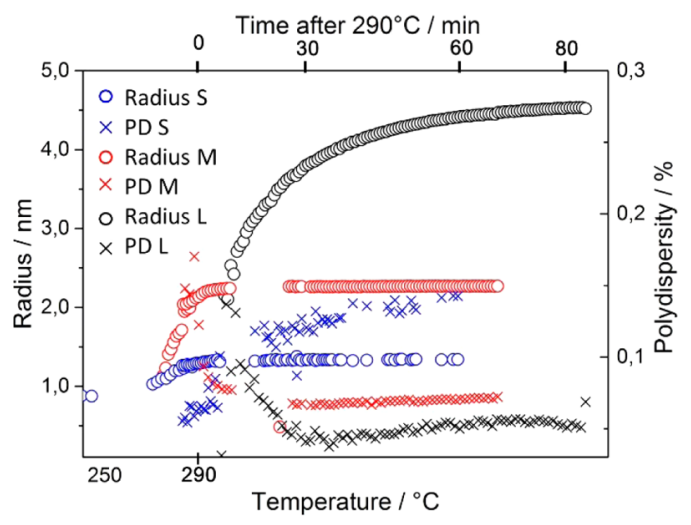

Figure 6. Time-evolution of radii and polydispersities for synthesis of NPs of different size. The size growth kinetics (circles) and polydispersity $\left(\mathrm{PD}\right.$, crosses) as a function of the $\mathrm{Fe}(\mathrm{CO})_{5} / \mathrm{OA}$ ratio for samples heated at $3 \mathrm{~K} / \mathrm{min}$. "Small" (blue), "Medium" (red), and "Large" (black) particles correspond to synthesis with $\mathrm{Fe}(\mathrm{CO})_{5} / \mathrm{OA}$ ratios in Table 1 .

NPs $\left(60+\right.$ minutes after reaching $\left.290^{\circ} \mathrm{C}\right)$. In contrast to the other sizes, the polydispersity of "Small" nanoparticles increases continuously during the growth phase and is also significantly higher than for the "Medium" and "Large" particles. It should however be noted that for such small particles very small differences in the number of atoms per particle lead to high polydispersity. Only one side maximum can be seen in the $q$ range of the experimental setup for "Small" particles, making the fit less robust and precise. The data gaps at $250-270{ }^{\circ} \mathrm{C}$ and at $\sim 15 \mathrm{~min}$ are due to an interruption of the experiment during which the beam had to be turned off.

The kinetics and temperature dependence of the Porod constant and Invariant as a function of the $\mathrm{Fe}(\mathrm{CO})_{5} / \mathrm{OA}$ ratio for samples heated at $3 \mathrm{~K} / \mathrm{min}$ are shown in Figure 7 . The Invariant and Porod constant both increase during the beginning of the heat-up phase irrespective of changes in the $\mathrm{Fe}(\mathrm{CO})_{5} / \mathrm{OA}$ ratio. The rate of formation of the iron oleate complex therefore seems to be independent of the precursor ratio and the oleic acid concentration. Thus, when the

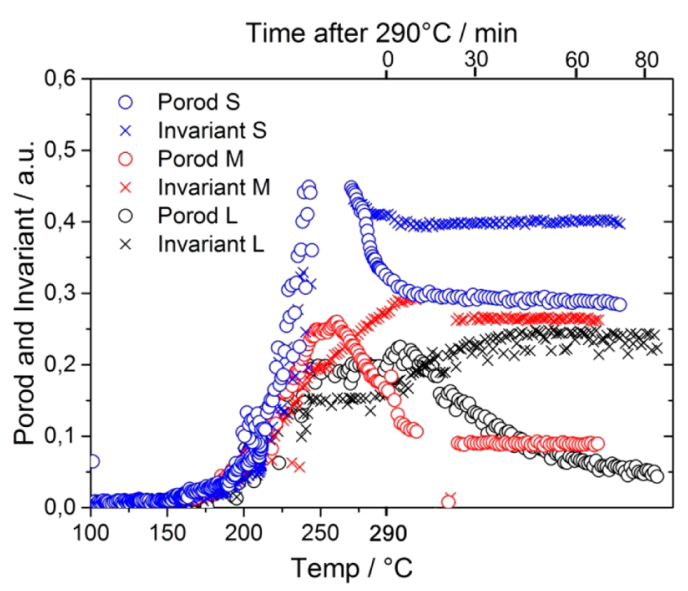

Figure 7. Time-evolution of Porod constant and Invariant for the synthesis of NPs of different size. The kinetics and temperature dependence of the Porod constant (circles) and Invariant (crosses) for different $\mathrm{Fe}(\mathrm{CO})_{5} / \mathrm{OA}$ ratios in samples heated at $3 \mathrm{~K} / \mathrm{min}$. "Small" (blue), "Medium" (red), and "Large" (black) particles correspond to synthesis with $\mathrm{Fe}(\mathrm{CO})_{5} / \mathrm{OA}$ ratios in Table 1 . 
temperature has reached $210-220{ }^{\circ} \mathrm{C}$, the $\mathrm{Fe}(\mathrm{CO})_{5}$ seems to have been fully decomposed to form the iron oleate complex. This is in very good agreement with the findings of Kwon et al. ${ }^{15}$ They spectroscopically measured the signal of the released $\mathrm{CO}$-ligand from $\mathrm{Fe}(\mathrm{CO})_{5}$ and found that the decomposition of $\mathrm{Fe}(\mathrm{CO})_{5}$ was completed at $\sim 210{ }^{\circ} \mathrm{C}$. A second phase that follows directly after the smooth increase in the Invariant and Porod constant observed up to $210{ }^{\circ} \mathrm{C}$ was observed but was difficult to distinguish from the $\mathrm{Fe}(\mathrm{CO})_{5}$ decomposition phase. This continued increase in the Invariant (signifying an increase in scattering material and contrast in the sample) above $210^{\circ} \mathrm{C}$ is strongly dependent on the $\mathrm{Fe}(\mathrm{CO})_{5} / \mathrm{OA}$ ratio. For higher $\mathrm{Fe}(\mathrm{CO})_{5} / \mathrm{OA}$ ratio (decreasing $\mathrm{OA}$ concentration), both the Invariant and the Porod constant show a steep increase to higher saturation values; conversely, saturation is observed at low values for the synthesis of "Large" particles with low $\mathrm{Fe}(\mathrm{CO})_{5} / \mathrm{OA}$ ratio.

The $\mathrm{Fe}(\mathrm{CO})_{5}$ concentration is the same for all samples. Thus, this difference in Porod and Invariant is not due to the different concentrations of iron oleate complex since all $\mathrm{Fe}(\mathrm{CO})_{5}$ is consumed in the reaction; it must be traced back to a difference in structure and contrast variation within the sample. A first interpretation is that there are more clusters/ particles (larger internal area) in this phase for a higher $\mathrm{Fe}(\mathrm{CO})_{5} / \mathrm{OA}$ ratio, leading to "Small" particles at the end of the synthesis. The reaction leading to this difference is extended over a longer time and therefore slower for "Small" particles compared to for "Large" particles.

The initial phases encompassing reactions of the precursors are followed by a plateau in both the Invariant and the Porod constant. The length of the plateau decreases with increased $\mathrm{Fe}(\mathrm{CO})_{5} / \mathrm{OA}$ ratio. Thus, it seems that the supersaturation condition (i.e., the end of the plateau) occurs at lower temperature for higher $\mathrm{Fe}(\mathrm{CO})_{5} / \mathrm{OA}$ ratio, although the iron oleate concentration is the same. This correlates with both a higher concentration of precursors (height of the plateau) and a lower excess of OA.

At the end of the plateau, burst nucleation is observed, at which point the Invariant decreases for all samples. Data are missing during the nucleation and initial growth of "Small" particles due to an air-bubble forming in the system, which makes it less evident in this case. It is difficult to compare if there is a difference in the increase in Invariant during the growth phase. The Invariant and the core growth start at different values, and it is difficult to extract data during the nucleation and initial growth phase for both the "Small" and the "Medium" data sets. The final value of the Invariant is, however, higher for higher $\mathrm{Fe}(\mathrm{CO})_{5} / \mathrm{OA}$ ratios (smaller $\mathrm{NPs}$ ). The Invariant thus scales inversely with particle size at a constant concentration of $\mathrm{Fe}(\mathrm{CO})_{5}$ precursor and presumably constant inorganic particle mass fraction.

As expected, the internal area, as described by the Porod constant, dramatically decreases for all ratios shortly after nucleation. It also scales inversely with $\mathrm{Fe}(\mathrm{CO})_{5} / \mathrm{OA}$ ratio and final particle size. The decrease in area and increase in scattering correlate well during the growth phase. The growth therefore clearly consumes precursor left after the nucleation step to bring down the concentration from supersaturation, and in this process the scattering contrast in the sample increases.

In summary, rather unexpectedly, the scattering signal of the precursor formed from the iron oleate complex is higher at higher $\mathrm{Fe}(\mathrm{CO})_{5} / \mathrm{OA}$ ratio (smaller NPs); this correlates with nucleation occurring at a lower temperature. Tentatively, a similar increase in scattering contrast occurs during growth for all particle sizes, but a larger reduction in internal surface area is indicated for "Small" particles than for "Large".

Effect of Heating Rate. Figure 8A shows the kinetics of the Porod constant and the Invariant for the NP formation process
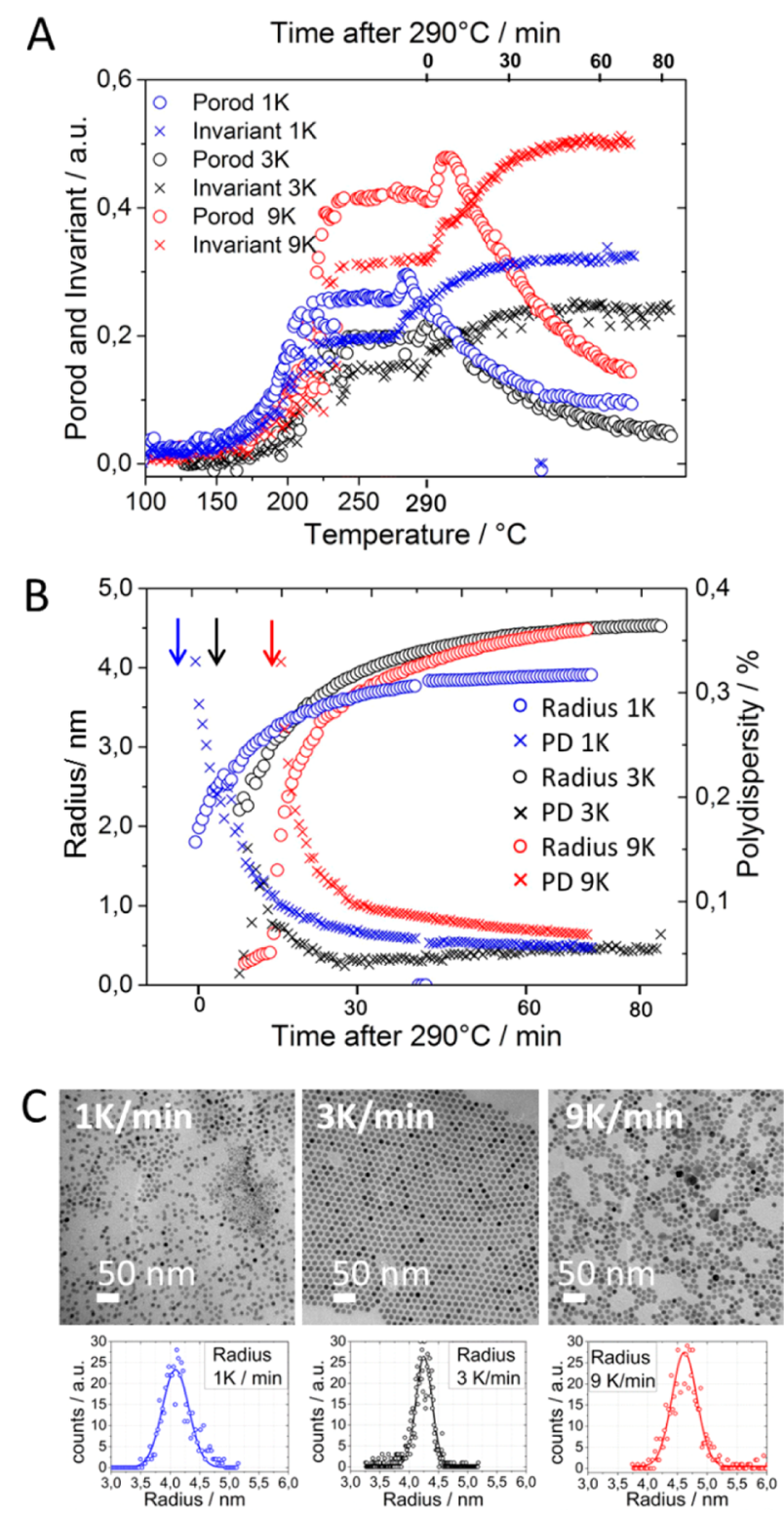

Figure 8. Synthesis of "Large" particles investigated as a function of heating rate. (A) Kinetics and temperature dependence of the Porod constant and Invariant. (B) Growth kinetics for radius and polydispersity of "Large" nanoparticles after nucleation. (C) Transmission electron micrographs of the particles after synthesis and the respective size distributions from image analysis for $1 \mathrm{~K} / \mathrm{min}, 3 \mathrm{~K} / \mathrm{min}$, and $9 \mathrm{~K} / \mathrm{min}$ heating rates.

as a function of the heating rate. At higher heating rates, there is a slight lag in the onset of the heat-up phase with respect to temperature. The plateau in the Porod constant and Invariant after formation of the complexes starts and ends with nucleation at a lower temperature for a lower heating rate and reaches higher values for both 1 and $9 \mathrm{~K} / \mathrm{min}$ compared to for the $3 \mathrm{~K} / \mathrm{min}$ heating rate. 
The burst nucleation and particle growth (indicated by arrows in Figure 8B) start before the final temperature $(T=$ $276{ }^{\circ} \mathrm{C}$ ) is reached at a heating rate of $1 \mathrm{~K} / \mathrm{min}$ but occur with a time lag at higher heating rates, that is, $0.5 \mathrm{~min}$ after the final temperature has been reached at a heating rate of $3 \mathrm{~K} / \mathrm{min}$ and $6.7 \mathrm{~min}$ after the final temperature has been reached for $9 \mathrm{~K} /$ min.

Although the kinetics do not change much with heating rate, its influence on the particle morphology is significant. Figure $8 \mathrm{C}$ shows representative transmission electron micrographs of the NPs synthesized at different heating rates and their respective size histograms. It is obvious that the NPs synthesized at 1 and $9 \mathrm{~K} / \mathrm{min}$ show a broader size distribution and that there are frequent occurrences of NPs with irregular shape. The formation of superlattices during assembly of OAcoated NPs on the TEM grid is a qualitative measure of low polydispersity and high sphericity. ${ }^{40}$ This is only observed for samples synthesized at $3 \mathrm{~K} / \mathrm{min}$ heating rate. NPs synthesized with 3 and $9 \mathrm{~K} / \mathrm{min}$ heating rates have similar final radii of $\sim 4.4$ $\mathrm{nm}$ measured by SAXS, and in agreement with a statistical analysis of the TEM data ( $\sim .3$ and $4.5 \mathrm{~nm}$ respectively, Figure $8 \mathrm{C}$ ). This size is slightly larger than the $\sim 3.9 \mathrm{~nm}$ average radius observed for the $1 \mathrm{~K} / \mathrm{min}$ heating rate. The polydispersity of the $3 \mathrm{~K} / \mathrm{min}$ synthesis seems lower immediately after nucleation and decreases much faster than for the other heating rates. A characteristic dip in polydispersity within the first $30 \mathrm{~min}$ was also observed at this heating rate, which led to an observed higher final polydispersity than at the minimum. This dip was not observed for the other heating rates, which demonstrated slower, monotonous decreases in polydispersity, eventually reaching a similar final value for all heating rates. However, although the final SAXS polydispersities are similar, it becomes clear from the TEM in Figure 8C that particles synthesized at $3 \mathrm{~K} / \mathrm{min}$ are significantly more spherical and uniform, to the level of showing self-crystallization, while the other heating rates produce particles with plenty of outlier particle sizes and inhomogeneous shapes.

\section{DISCUSSION}

Our high time resolution SAXS data and corresponding TEM images of the nanoparticle synthesis confirm the previous model for the core synthesis. ${ }^{14,42}$ However, they also provide crucial additional detail to the process, most importantly concerning the formation of precursors and the nucleation step. By studying the Porod constant and Invariant changes over time, we clearly identify six phases of the synthesis that are mapped and illustrated in Figure 9: (i) heat-up lag phase; (ii) decomposition of the precursors to form iron oleate; (iii) formation of clusters or prenuclei (precursors); (iv) a second heating lag phase; (v) burst nucleation; and (vi) core growth with narrowing of the size distribution. The lengths of the different phases, in particular the second lag phase (iv) and the growth phase (vi), vary with the synthesis conditions. These differences will be discussed in detail below.

Structure Formation during Initial Heat-up Phase: Formation of Intermediate Products. The fact that the iron oleate complexes and precursor states are detectable through both the Invariant and the Porod constant implies that the complex fluid has structure, since otherwise the fluid will not contain any phase boundaries with sufficient scattering contrast. We emphasize that the SAXS signal during the heat-up phase is devoid of a distinct particle signal and WAXS is similarly indicating the absence of crystalline material in the sample. This

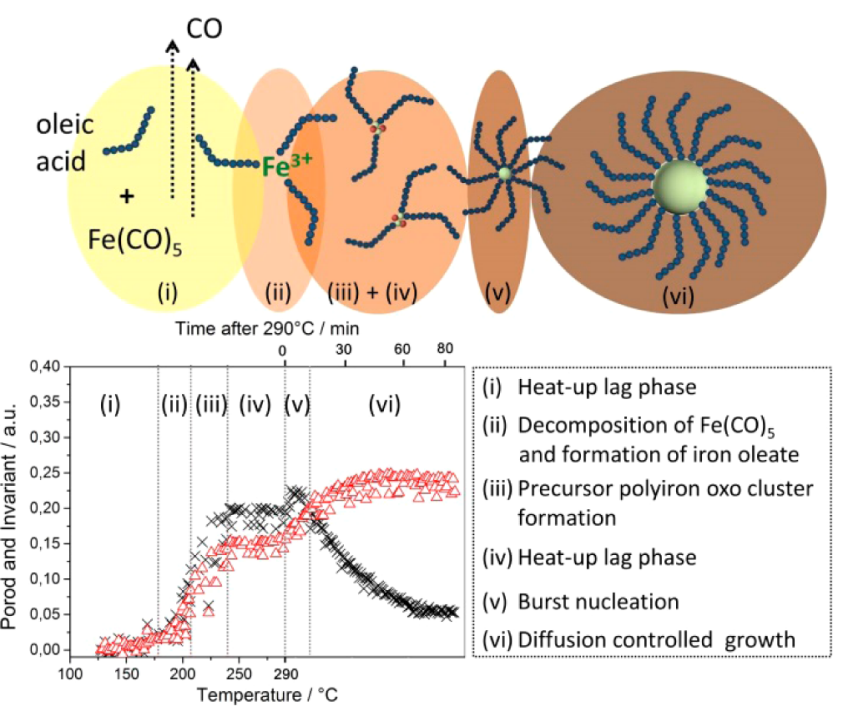

Figure 9. Distinct phases identified in the synthesis of iron oxide NPs via heat-up thermal decomposition. Six growth phases are identified, exemplified in the data plot of the Porod and Invariant for the synthesis of "Large" particles.

suggests that the most likely origin of the increased scattering lies in micellar structures of complexes or precursor with an oleate/oleic acid shell. These are formed in the dioctyl ether solvent with additional dissolved $\mathrm{OA}$ in the continuous phase.

The most pronounced difference between samples was observed when the $\mathrm{Fe}(\mathrm{CO})_{5} / \mathrm{OA}$ ratio was varied with constant concentration of $\mathrm{Fe}(\mathrm{CO})_{5}$. The difference between the samples is evident already during the heat-up phase. During this phase the different samples start to deviate in the scattering signal at temperatures slightly above $210^{\circ} \mathrm{C}$. Up to this temperature, the different samples show very similar rates of increase in Invariant and Porod constant. This temperature range agrees well with the temperature range within which in situ infrared spectroscopy has revealed decomposition of $\mathrm{Fe}(\mathrm{CO})_{5}$ through the release of $\mathrm{CO}$ and a strong reduction of the signal of the carboxyl group of free $\mathrm{OA}$, indicating that the formation of the iron oleate complex is completed at that temperature. ${ }^{15}$

The Invariant and the internal area drastically increase above $210{ }^{\circ} \mathrm{C}$ for "Small" particles, while they increase at a slower rate for "Medium" and "Large" cores. This strongly indicates that above $\sim 210^{\circ} \mathrm{C}$ the iron oleate complex undergoes a reaction to a different precursor state for the nanoparticle formation. Kwon et al. have also suggested that such a conversion occurs, hypothetically to polyiron oxo clusters. In their in situ infrared spectroscopy study, the onset of the signal of the metal binding carboxyl groups is at $\sim 210{ }^{\circ} \mathrm{C}$, and they suggest that an intermediate species is formed between the decomposition of iron oleate and the reaching of the final temperature. ${ }^{15}$ The presence of this intermediate species was additionally supported in follow-up work by the same group, in which MALDI-ToF-MS and TEM were used to resolve small particles in the sample before nucleation occurred. ${ }^{43}$ These particles possessed neither the same magnetic properties nor crystallinity as superparamagnetic iron oxide nanoparticles after nucleation. However, they were shown to possess a strongly bound oleate shell. Our size-sensitive and highly time-resolved study using SAXS further strongly supports the formation of dense clusters as intermediates since the scattering signal continues to increase after the formation of iron oleate. Furthermore, we observe that 
the evolution of the signal differs as a function of the $\mathrm{Fe}(\mathrm{CO})_{5} /$ $\mathrm{OA}$ ratio. The kinetics of formation of scattering centers suggests that the $\mathrm{Fe}(\mathrm{CO})_{5}$ is fully decomposed and the pool of iron oleate fully formed already at $\sim 210{ }^{\circ} \mathrm{C}$. The scattering signal is consistent with that these complexes form small but diffuse (presumably micellar) aggregates from which precursor clusters can form. Furthermore, the formation of the precursor clusters proceeds over a short time-window at temperatures slightly above $210^{\circ} \mathrm{C}$. The formation of clusters already at this low temperature agrees with the results of Bronstein et al. ${ }^{44}$ and Park et al. ${ }^{6}$ who showed that the dissociation of the first oleate group from the iron oleate complex upon heating starts at about $200{ }^{\circ} \mathrm{C}$. The decomposition of the iron oleate complexes in aggregates to form more strongly scattering clusters is in all cases complete before $250{ }^{\circ} \mathrm{C}$, or $\sim 15$ min after starting, for a heating rate of $3 \mathrm{~K} / \mathrm{min}$, indicated by the onset of the pronounced plateau in the plot of the Porod constant and Invariant. This is in disagreement with the findings of Bronstein et al. ${ }^{44}$ and Park et al. ${ }^{6}$ They suggest the dissociation of the two remaining coordinated oleate groups at a temperature of 300 ${ }^{\circ} \mathrm{C}$. However, one should keep in mind that their iron oleate complexes were synthesized ex situ. It has been shown that oleate complexes show very different stabilities depending on their synthesis parameters and handling, ${ }^{44}$ presumably leading to different levels of purity.

It also seems a higher concentration of precursor clusters is formed at higher $\mathrm{Fe}(\mathrm{CO})_{5} / \mathrm{OA}$ ratios, that is, at lower excess of $\mathrm{OA}$ and constant concentration of $\mathrm{Fe}(\mathrm{CO})_{5}$. Oleate coordinated to the cluster surface will act as a shell protecting the clusters from interaction, but they simultaneously can contribute to the total scattering intensity since the organized molecules in the shell produce a small but significant scattering contrast to the surrounding solution. The latter can explain that the "Small" cores not only have a higher internal area, but also have a highly increased overall scattering signal (Invariant); the number of clusters formed is higher, which increases the total amount of dense, structured OA around clusters in the sample. Our data thus show that the cluster composition varies with the synthesis conditions $\left(\mathrm{Fe}(\mathrm{CO})_{5} / \mathrm{OA}\right.$ ratio $)$ and that the oleate packing density on the cluster surface is not constant as suggested from a fit to particle growth for a single set of conditions. ${ }^{43}$ The fact that the stoichiometric ratio of precursors can influence the ligation of primary particles consumed during nucleation and thereby affect diffusion limited nanoparticle growth is not unprecedented; it has been demonstrated by Angelopoulus and co-workers experimentally and theoretically for diffusion limited growth of $\mathrm{Pt}$ nanoparticles. ${ }^{22}$ Importantly, the clusters are amorphous (no crystallinity is detected) and too small or polydisperse to be called particles since a form factor cannot be seen in the scattering signal when only this precursor state is present.

The heating rate affects the cluster formation step, with both high $(9 \mathrm{~K} / \mathrm{min})$ and low $(1 \mathrm{~K} / \mathrm{min})$ heating rates leading to a higher Invariant plateau proportional to a higher internal area and somewhat higher Porod constant proportional to the scattering contrast in the sample (Figure $8 \mathrm{~A}$ ). For the slow 1 $\mathrm{K} / \mathrm{min}$ heating rate, this again seems to correspond to a larger number of clusters being formed. The biggest difference is observed for the high $9 \mathrm{~K} / \mathrm{min}$ heating rate. It is unclear if this also is due to the formation of a larger number of smaller clusters since the correlation of this increase in signal with final nanoparticle size is different than for the other samples, as discussed below.
Nucleation. Classical nucleation theory ${ }^{14,45}$ describes the crystal nucleation rate per unit volume as

$$
J_{\mathrm{N}}=B_{\mathrm{N}} \exp \left(-\frac{\Delta G^{\mathrm{N}}}{R T}\right)
$$

where the activation energy for homogeneous nucleation is described by

$$
\Delta G^{\mathrm{N}}=\frac{16 \pi \gamma^{3} V_{\mathrm{m}}^{2}}{3(\Delta \mu)^{2}}
$$

and $B_{\mathrm{N}}$ is a pre-exponential factor depending on many factors regarding the precursor species, $\gamma$ is the surface tension, and $V_{\mathrm{m}}$ is the molar volume of the solid. $\Delta \mu$ is the difference in chemical potential between the solid particle and the free monomer states, which for oversaturation can be approximated as

$$
\Delta \mu=R T \ln S
$$

where the oversaturation $S$ is the ratio between the current and the equilibrium concentration of monomer.

A higher number of clusters (precursors) therefore contributes to lower the barrier and strongly contributes to increase the rate of nucleation, which can now occur at a lower temperature as shown by our data. A higher $\mathrm{Fe}(\mathrm{CO})_{5} / \mathrm{OA}$ ratio corresponds to a higher concentration of clusters, a higher supersaturation ratio, and therefore synthesis of "Small" NPs. We also note that the high $\mathrm{Fe}(\mathrm{CO})_{5} / \mathrm{OA}$ ratio with more and smaller clusters suggests a thinner shell around clusters for "Small" core synthesis and an increase in $\Delta \mu$, which also contributes to a lowering of the temperature at which supersaturation leads to burst nucleation. For "Large" cores, the lower concentration of precursor clusters to begin with and the presumably denser shell leading to better stabilization of larger clusters translates into a long lag phase during further heating; no structural change occurs in the sample due to the larger energetic barrier to reaction of the dense shell. It is clear from eq 8 that the nucleation rate is very sensitive to both temperature and difference in chemical potential; this enables the separation of nucleation and growth as well as to control the nucleation condition.

It previously has been suggested that decomposition of $\mathrm{Fe}(\mathrm{CO})_{5}$ proceeds over a large temperature range during the heat-up phase. ${ }^{6}$ Our results indicate that this depends on the $\mathrm{Fe}(\mathrm{CO})_{5} / \mathrm{OA}$ ratio since only at lower $\mathrm{Fe}(\mathrm{CO})_{5} / \mathrm{OA}$ ratios structural change is a observed in the temperature range between formation of the iron oleate complex and reflux. Furthermore, the structural change observed in this temperature range for the synthesis of "Small" nanoparticles seems to correspond directly to the burst nucleation and growth occurring at lower temperature. We therefore propose that especially for the synthesis of "Large" cores by this route there can be, and is, a significant separation (in temperature and time) between formation of precursor clusters and nucleation.

Nucleation will occur as soon as the temperature is high enough to overcome the energy barrier of homogeneous nucleation from the precursor. ${ }^{13}$ Burst nucleation is achieved if a supersaturated solution of precursor is suddenly heated above the nucleation temperature. The peak in the Porod constant (and to a smaller extent the Invariant) found in phase (v), which lasts for only a few minutes, is evidence that the burst nucleation occurs. The maximum inner surface area is obtained during the nucleation step. The fact that the Porod constant of 
the sample containing nuclei is higher than that containing only precursor clusters is likely due to the diffuse and unstructured interface expected for the clusters; the magnitude of the Porod constant depends on the contrast at this interface in addition to on the total inner surface area for a multiphase system.

The early burst nucleation at temperatures below the reflux temperature for "Small" cores can be problematic for synthesis of monodisperse nanoparticles. If precursors are still formed as nucleation takes place, there might be continuous nucleation, leading to the nucleation and growth phases that are not separated. This could partly explain why "Small" cores not only have a much higher relative polydispersity than "Large" particles, but also a less regular spherical morphology, as evident from TEM data (Figure S1). A possible suggestion based on this could be to use ligands with even higher density and surface affinity for the synthesis of small cores.

The heating rate seems to affect the nucleation step as well as the formation of clusters (cf. Figure 8). The nucleation takes place at a delay after the reflux temperature has been reached when the heating rate is high $(9 \mathrm{~K} / \mathrm{min})$. While the direct cause for this lag is not clear, this could, for example, relate to heating gradients in the sample as the temperature is raised fast at the periphery of the reaction vessel, or a lower accumulated probability that nucleation has initiated after the shorter total time spent at sufficiently high temperature. Such temperature lags or gradients imply that a more heterogeneous nucleation is likely at lower heating rates. This hypothesis is also supported by the TEM of final nanoparticle distributions; most of the 9 $\mathrm{K} / \mathrm{min}$ sample has a size and size distribution similar to the 3 $\mathrm{K} / \mathrm{min}$ (optimal) heating rate, but there is also a significant fraction of larger and nonspherical particles present. It is likely that this difference can be traced back to nonuniform nucleation. Despite that the cluster formation step and nucleation step seem well separated, the nucleation and growth steps are likely less separated. However, we note that there is a large difference also in the preceding cluster formation step, where a much larger internal area is evident for the $9 \mathrm{~K} / \mathrm{min}$ compared to the $3 \mathrm{~K} / \mathrm{min}$ sample, but uniquely in this case it is obviously not correlated to a larger number of small cores after completed growth, but to higher final polydispersity and higher number of irregular cores.

The most interesting effect is observed for the lowest $1 \mathrm{~K} /$ min heating rate. For this heating rate, nucleation starts at a temperature significantly below the reflux temperature. Again, the effect seems to be a more heterogeneous nucleation phase, where cores nucleate and start growing at different temperatures before stable conditions are reached. Presumably, nucleation is spread out in time and temperature due to a finite nucleation probability also at lower temperature given sufficient time.

Comparison of Size-Focused Growth of "Small" and "Large" Cores. Nucleation is followed by what is presumed to be diffusion-controlled growth (phase (vi)). A hallmark of diffusion controlled growth is that it allows size focusing to occur, that is, the polydispersity can narrow to below the typical $10 \%$ observed for separated burst nucleation and growth. ${ }^{46}$ Our data in Figure 6 suggest that size focusing due to diffusion controlled core growth is evident for both larger particle sizes. The synthesis of these larger cores is accomplished by choosing a low $\mathrm{Fe}(\mathrm{CO})_{5} / \mathrm{OA}$ ratio. The polydispersity shown in Figure 6 decreases very rapidly after the burst nucleation. For large cores (low $\mathrm{Fe}(\mathrm{CO})_{5} / \mathrm{OA}$ ratio), size focusing was observed to occur after nucleation as a decrease in variance of the fitted particle distribution. The initial strong decrease is followed by a slight increase in polydispersity. In contrast, for the "Small" cores (high $\mathrm{Fe}(\mathrm{CO})_{5} / \mathrm{OA}$ ratio), a continuous increase in polydispersity was observed from the beginning. This can however be an artifact due to that the nucleation and initial part of the growth is missing in the data due to an interruption of the experiment by clogging.

The origin of the small increase in polydispersity after the initial size focusing at $40 \mathrm{~min}$ reaction time for "Large" cores is unclear since Ostwald ripening should not occur for diffusion controlled growth. Although this increase is marginal at only $\sim 2 \%$, it could suggest that quenching the reaction at an optimal time during growth could further reduce the polydispersity. The continuous increase in polydispersity of "Small" cores during the growth phase could be related to the more inhomogeneous nucleation for "Small" cores. As described above, for these nanoparticles, the precursor formation and nucleation phases have merged and might cause heterogeneity also in the growth phase since the nucleation phase is short. An alternative interpretation can be based on that our data show a higher concentration of not only nuclei, but also precursor clusters for "Small" cores. For mass conservation reasons, these must be smaller as well as more reactive, which is supported by the kinetic data. The larger amount and higher reactivity of precursor clusters could presumably lead to less controlled nucleation and growth and affect both polydispersity and nonuniform morphology.

Influence of Heating Rate on Size Focusing. As seen in Figure $8 \mathrm{~B}$, the core growth is slower, and polydispersity decreases slower and more continuously for the $1 \mathrm{~K} / \mathrm{min}$ heating rate than for the $3 \mathrm{~K} / \mathrm{min}$ sample. At $3 \mathrm{~K} / \mathrm{min}$ heating rate, very strong size focusing is observed during the early growth phase. The final average core size is smaller at the slow heating rate than at the two higher rates. Although the polydispersity estimated from the fits is not much different to that of the optimal $3 \mathrm{~K} / \mathrm{min}$ heating rate sample, TEM inspection of the $1 \mathrm{~K} / \mathrm{min}$ sample clearly demonstrates less uniform cores in terms of size (Figure 8C). A slower growth rate, starting at a lower temperature with nucleation less focused in time compared to the optimal $3 \mathrm{~K} / \mathrm{min}$ heating rate, thus seems to be the consequence of a (too) slow heating rate; this produces more faceted and therefore not only potentially more crystalline, but also more heterogeneous cores.

The average polydispersity after growth is not much higher for the $9 \mathrm{~K} / \mathrm{min}$ sample, and it is still converging at the point of finishing the experiment. The cores are then still growing, albeit slowly. TEM investigations, however, show that larger cores, potentially growing for longer time, are present in the $9 \mathrm{~K} / \mathrm{min}$ sample but not in the $3 \mathrm{~K} / \mathrm{min}$ sample (Figure $8 \mathrm{C}$ ). These cores will never converge to the high uniformity observed at the intermediate heating rate even if the growth phase had been prolonged.

\section{CONCLUSIONS}

We have demonstrated a setup suitable for following the growth of monodisperse nanoparticles using the heat-up and thermal decomposition method at the highest time resolution to date using in situ SAXS and WAXS. This approach could in the future be combined to include, for example, UV-vis/IR spectroscopies that were previously used to provide chemical information on the growth stages during nanoparticle synthesis.

While the time evolution of geometrical core size growth and polydispersity could be fitted with second resolution after 
nanoparticle nucleation, the most important new insights were obtained by analyzing the time evolution of the Porod constant and the Invariant before nucleation occurred. These parameters yield insight into changes in inhomogeneity and the creation of strongly scattering material within the sample without resorting to a detailed a priori assumed model for fitting of data. Using this analysis, we found that optimal synthesis of monodisperse iron oxide nanoparticles is composed of six well-defined phases in agreement with, but providing increased detail to, current models used to describe heat-up thermal decomposition synthesis of (iron oxide) nanoparticles. These phases are mostly well separated but include intermeshed phases for iron oleate complex formation and precursor (polyiron oxo) cluster formation. Strikingly, a clear separation of the phases of cluster formation and burst nucleation at supersaturation by an additional heat-up lag phase was found for the synthesis of the most monodisperse and uniform nanoparticles. Another novel insight of this analysis of separated phases was the correlation of a difference in the number, size, and density of precursor clusters formed during heat-up with the number of nuclei formed during the burst nucleation phase that controls the final particle size. This could be exquisitely controlled by the choice of $\mathrm{Fe}(\mathrm{CO})_{5} / \mathrm{OA}$ ratio, but it was also affected by the heating rate.

The highest morphological (spherical) uniformity and lowest relative polydispersity was achieved for synthesis of large nanoparticles (targeted by a low $\mathrm{Fe}(\mathrm{CO})_{5} / \mathrm{OA}$ ratio) at a heating rate of $3 \mathrm{~K} / \mathrm{min}$. These conditions seemed to achieve a clear separation of the kinetic steps and a sufficiently slow growth to form spherical particles with the lowest polydispersity. The assumed optimal conditions for producing small nanoparticles, however, seemed to lead to a fast growth rate and smaller than expected crystal size, which indicated that further optimization by tuning precursor ratios and heating rate could be beneficial for performance and that quenching of the synthesis at an optimal time could further reduce polydispersity.

\section{ASSOCIATED CONTENT}

\section{S Supporting Information}

The Supporting Information is available free of charge on the ACS Publications website at DOI: 10.1021/acs.chemmater.7b01207.

Additional data on all reactions; TEM micrographs; electron diffraction patterns; WAXS patterns; detailed experimental information; images of experimental setup; additional calculations on flux normalization (PDF)

\section{AUTHOR INFORMATION}

\section{Corresponding Authors}

*E-mail: Erik.Reimhult@boku.ac.at.

*E-mail: Helga.Lichtenegger@boku.ac.at.

\section{ORCID}

E. Reimhult: 0000-0003-1417-5576

\section{Present Address}

${ }^{\mathbb{I}}$ Tilman A. Grünewald, Beamline ID13, X-ray nanoprobe group (XNP), ESRF - The European Synchrotron, 71 Avenue des Martyrs, CS 40220, 38043 Grenoble Cedex 9, France.

\section{Author Contributions}

${ }^{\perp}$ A.L. and T.A.G. contributed equally to this work. The work was planned by E.R., H.L., P.D.J.v.O., R.Z., A.L., and T.A.G.;
NP synthesis and TEM were done by A.L.; SAXS measurements were performed by T.A.G., A.L., and H.R.; setup was built by T.A.G. and P.D.J.v.O.; SAXS data evaluation was done by T.A.G.; and the manuscript was written by E.R., A.L., T.A.G., and H.L.

\section{Funding}

The research leading to these results received funding from the European Research Council under the European Union's Seventh Framework Program (FP/2007-2013)/ERC Grant Agreement No. 310034.

Notes

The authors declare no competing financial interest.

\section{ACKNOWLEDGMENTS}

We thank the Austrian SAXS beamline at Elettra Synchrotone Trieste.

\section{REFERENCES}

(1) Farmer, S. C.; Patten, T. E. Photoluminescent Polymer/Quantum Dot Composite Nanoparticles. Chem. Mater. 2001, 13, 3920-3926.

(2) Sangermano, M.; Messori, M. Scratch Resistance Enhancement of Polymer Coatings. Macromol. Mater. Eng. 2010, 295, 603-612.

(3) Smith, B. R.; Gambhir, S. S. Nanomaterials for In Vivo Imaging. Chem. Rev. 2017, 117, 901-986.

(4) Petros, R. A.; DeSimone, J. M. Strategies in the design of nanoparticles for therapeutic applications. Nat. Rev. Drug Discovery 2010, 9, 615-627.

(5) Morgan, S. E.; Havelka, K. O.; Lochhead, R. Y.; Chemistry, A. C. S. D. o. P. Cosmetic Nanotechnology: Polymers and Colloids in Cosmetics; American Chemical Society, 2007.

(6) Park, J.; An, K.; Hwang, Y.; Park, J.-G.; Noh, H.-J.; Kim, J.-Y.; Park, J.-H.; Hwang, N.-M.; Hyeon, T. Ultra-large-scale syntheses of monodisperse nanocrystals. Nat. Mater. 2004, 3, 891-895.

(7) Park, J.; Lee, E.; Hwang, N.-M.; Kang, M.; Kim, S. C.; Hwang, Y.; Park, J.-G.; Noh, H.-J.; Kim, J.-Y.; Park, J.-H.; Hyeon, T. Onenanometer-scale size-controlled synthesis of monodisperse magnetic iron oxide nanoparticles. Angew. Chem., Int. Ed. 2005, 44, 2872-2877.

(8) Sun, S.; Zeng, H. Size-Controlled Synthesis of Magnetite Nanoparticles. J. Am. Chem. Soc. 2002, 124, 8204-8205.

(9) Yu, W. W.; Falkner, J. C.; Yavuz, C. T.; Colvin, V. L. Synthesis of monodisperse iron oxide nanocrystals by thermal decomposition of iron carboxylate salts. Chem. Commun. 2004, 2306-2307.

(10) Xu, Z.; Shen, C.; Hou, Y.; Gao, H.; Sun, S. Oleylamine as both reducing agent and stabilizer in a facile synthesis of magnetite nanoparticles. Chem. Mater. 2009, 21, 1778-1780.

(11) Park, J.; Joo, J.; Kwon, S. G.; Jang, Y.; Hyeon, T. Synthesis of monodisperse spherical nanocrystals. Angew. Chem., Int. Ed. 2007, 46, 4630-4660.

(12) Kovalenko, M. V.; Bodnarchuk, M. I.; Lechner, R. T.; Hesser, G.; Schaeffler, F.; Heiss, W. Fatty Acid Salts as Stabilizers in Size- and Shape-Controlled Nanocrystal Synthesis: The Case of Inverse Spinel Iron Oxide. J. Am. Chem. Soc. 2007, 129, 6352-6353.

(13) LaMer, V. K.; Dinegar, R. H. Theory, Production and Mechanism of Formation of Monodispersed Hydrosols. J. Am. Chem. Soc. 1950, 72, 4847-4854.

(14) Talapin, D. V.; Rogach, A. L.; Haase, M.; Weller, H. Evolution of an Ensemble of Nanoparticles in a Colloidal Solution: Theoretical Study. J. Phys. Chem. B 2001, 105, 12278-12285.

(15) Kwon, S. G.; Hyeon, T. Colloidal Chemical Synthesis and Formation Kinetics of Uniformly Sized Nanocrystals of Metals, Oxides, and Chalcogenides. Acc. Chem. Res. 2008, 41, 1696-1709.

(16) Lee, N.; Hyeon, T. Designed synthesis of uniformly sized iron oxide nanoparticles for efficient magnetic resonance imaging contrast agents. Chem. Soc. Rev. 2012, 41, 2575-2589.

(17) Bronstein, L. M.; Atkinson, J. E.; Malyutin, A. G.; Kidwai, F.; Stein, B. D.; Morgan, D. G.; Perry, J. M.; Karty, J. A. Nanoparticles by 
Decomposition of Long Chain Iron Carboxylates: From Spheres to Stars and Cubes. Langmuir 2011, 27, 3044-3050.

(18) Kwon, S. G.; Piao, Y.; Park, J.; Angappane, S.; Jo, Y.; Hwang, N.M.; Park, J.-G.; Hyeon, T. Kinetics of Monodisperse Iron Oxide Nanocrystal Formation by "Heating-Up" Process. J. Am. Chem. Soc. 2007, 129, 12571-12584.

(19) Kwon, S. G.; Hyeon, T. Colloidal Chemical Synthesis and Formation Kinetics of Uniformly Sized Nanocrystals of Metals, Oxides, and Chalcogenides. Acc. Chem. Res. 2008, 41, 1696-1709.

(20) Lifshitz, I. M.; Slyozov, V. V. The kinetics of precipitation from supersaturated solid solutions. J. Phys. Chem. Solids 1961, 19, 35-50.

(21) Wagner, C. Theory of precipitate change by redissolution. $Z$. Elektrochem. Angew. Phys. Chem. 1961, 65, 581-91.

(22) St. John, S.; Nan, Z.; Hu, N.; Schaefer, D. W.; Angelopoulos, A. P. A nanoscale-modified LaMer model for particle synthesis from inorganic tin-platinum complexes. J. Mater. Chem. A 2013, 1, 89038916.

(23) Talapin, D. V.; Rogach, A. L.; Shevchenko, E. V.; Kornowski, A.; Haase, M.; Weller, H. Dynamic Distribution of Growth Rates within the Ensembles of Colloidal II-VI and III-V Semiconductor Nanocrystals as a Factor Governing Their Photoluminescence Efficiency. J. Am. Chem. Soc. 2002, 124, 5782-5790.

(24) Luigjes, B.; Woudenberg, S. M. C.; de Groot, R.; Meeldijk, J. D.; Torres Galvis, H. M.; de Jong, K. P.; Philipse, A. P.; Erne, B. H. Erne, B. H., Diverging Geometric and Magnetic Size Distributions of Iron Oxide Nanocrystals. J. Phys. Chem. C 2011, 115, 14598-14605.

(25) Gatteschi, D.; Caneschi, A.; Pardi, L.; Sessoli, R. Large clusters of metal ions: the transition from molecular to bulk magnets. Science 1994, 265, 1054-1058.

(26) Hyeon, T.; Lee, S. S.; Park, J.; Chung, Y.; Na, H. B. Synthesis of Highly Crystalline and Monodisperse Maghemite Nanocrystallites without a Size-Selection Process. J. Am. Chem. Soc. 2001, 123, 1279812801 .

(27) Yi, Z.; Dumee, L. F.; Garvey, C. J.; Feng, C.; She, F.; Rookes, J. E.; Mudie, S.; Cahill, D. M.; Kong, L. A New Insight into Growth Mechanism and Kinetics of Mesoporous Silica Nanoparticles by in Situ Small Angle X-ray Scattering. Langmuir 2015, 31, 8478-8487.

(28) Liu, J.; Pancera, S.; Boyko, V.; Shukla, A.; Narayanan, T.; Huber, K. Evaluation of the Particle Growth of Amorphous Calcium Carbonate in Water by Means of the Porod Invariant from SAXS. Langmuir 2010, 26, 17405-17412.

(29) Forny-Le Follotec, A.; Glatter, O.; Pezron, I.; Barre, L.; Noik, C.; Dalmazzone, C.; Metlas-Komunjer, L. Characterization of Micelles of Small Triblock Copolymer by Small-Angle Scattering. Macromolecules 2012, 45, 2874-2881.

(30) Li, T.; Senesi, A. J.; Lee, B. Small Angle X-ray Scattering for Nanoparticle Research. Chem. Rev. 2016, 116, 11128-11180.

(31) de Mello Donega, C.; Liljeroth, P.; Vanmaekelbergh, D. Physicochemical evaluation of the hot-injection method, a synthesis route for monodisperse nanocrystals. Small 2005, 1, 1152-1162.

(32) Amenitsch, H.; Rappolt, M.; Kriechbaum, M.; Mio, H.; Laggner, P.; Bernstorff, S. First performance assessment of the small-angle X-ray scattering beamline at ELETTRA. J. Synchrotron Radiat. 1998, 5, 506508.

(33) Langford, J. I.; Wilson, A. J. C. Scherrer after sixty years: A survey and some new results in the determination of crystallite size. $J$. Appl. Crystallogr. 1978, 11, 102-113.

(34) Kotlarchyk, M.; Stephens, R. B.; Huang, J. S. Study of Schultz distribution to model polydispersity of microemulsion droplets. J. Phys. Chem. 1988, 92, 1533-1538.

(35) Levenberg, K. A Method for the Solution of Certain Non-Linear Problems in Least Squares. Q. Appl. Math. 1944, 2, 164-168.

(36) Marquardt, D. W. An Algorithm for Least-Squares Estimation of Nonlinear Parameters. J. Soc. Ind. Appl. Math. 1963, 11, 431-441.

(37) Glatter, O.; Kratky, O. Small Angle X-Ray Scattering.; Academic Press: London, 1982.

(38) Rayleigh, L. The Incidence of Light upon a Transparent Sphere of Dimensions Comparable with the Wave-Length. Proc. R. Soc. London, Ser. A 1910, 84, 25-46.
(39) Mondini, S.; Ferretti, A. M.; Puglisi, A.; Ponti, A. Pebbles and PebbleJuggler: software for accurate, unbiased, and fast measurement and analysis of nanoparticle morphology from transmission electron microscopy (TEM) micrographs. Nanoscale 2012, 4, 5356-5372.

(40) Pusey, P. N.; Zaccarelli, E.; Valeriani, C.; Sanz, E.; Poon, W. C. K.; Cates, M. E. Hard spheres: crystallization and glass formation. Philos. Trans. R. Soc., A 2009, 367, 4993-5011.

(41) Finger, L. W.; Hazen, R. M.; Hofmeister, A. M. High-pressure crystal chemistry of spinel (MgAl2O4) and magnetite ( $\mathrm{Fe} 3 \mathrm{O} 4)$ : comparisons with silicate spinels. Phys. Chem. Miner. 1986, 13, 215220 .

(42) Kwon, S. G.; Hyeon, T. Formation Mechanisms of Uniform Nanocrystals via Hot-Injection and Heat-Up Methods. Small 2011, 7, 2685-2702.

(43) Kim, B. H.; Shin, K.; Kwon, S. G.; Jang, Y.; Lee, H.-S.; Lee, H.; Jun, S. W.; Lee, J.; Han, S. Y.; Yim, Y.-H.; Kim, D.-H.; Hyeon, T. Sizing by Weighing: Characterizing Sizes of Ultrasmall-Sized Iron Oxide Nanocrystals Using MALDI-TOF Mass Spectrometry. J. Am. Chem. Soc. 2013, 135, 2407-2410.

(44) Bronstein, L. M.; Huang, X.; Retrum, J.; Schmucker, A.; Pink, M.; Stein, B. D.; Dragnea, B. Influence of iron oleate complex structure on iron oxide nanoparticle formation. Chem. Mater. 2007, 19, 36243632 .

(45) Shevchenko, E. V.; Talapin, D. V.; Schnablegger, H.; Kornowski, A.; Festin, O.; Svedlindh, P.; Haase, M.; Weller, H. Study of Nucleation and Growth in the Organometallic Synthesis of Magnetic Alloy Nanocrystals: The Role of Nucleation Rate in Size Control of CoPt3 Nanocrystals. J. Am. Chem. Soc. 2003, 125, 9090-9101.

(46) Peng, X.; Wickham, J.; Alivisatos, A. P. Kinetics of II-VI and IIIV Colloidal Semiconductor Nanocrystal Growth: "Focusing" of Size Distributions. J. Am. Chem. Soc. 1998, 120, 5343-5344. 